\title{
Predictive factors of the attack efficacy: the case of the Brazilian women's Volleyball League champion team
}

\section{Fatores preditivos da eficácia do ataque: o caso da equipe campeã da superliga feminina}

\author{
Augusto Cézar Rodrigues Rocha ${ }^{1}$ \\ (D) https://orcid.org/0000-0003-3583-5676 \\ Auro Barreiros Freire ${ }^{2}$ \\ (D) https://orcid.org/0000-0001-5198-9363 \\ Leonardo Rodrigues Martins ${ }^{1}$ \\ (D) https://orcid.org/0000-0001-8224-3177 \\ Mariana Pereira Maia \\ (D) https://orcid.org/0000-0003-4763-4994 \\ Juracy da Silva Guimarães \\ (D) https://orcid.org/0000-0002-9928-3869 \\ Herbert Ugrinowitsch ${ }^{3}$ \\ (D) https://orcid.org/ 0000-0003-0317-1940 \\ Henrique de Oliveira Castro ${ }^{4}$ \\ (D) https://orcid.org/0000-0002-0545-164X \\ Gustavo De Conti Teixeira Costa \\ (D) https://orcid.org/0000-0003-0911-8753
}

Abstract - The aim was to identify the predictive factors of the attack efficacy of the Brazilian women's League Champion team according to the level of performance of the opposing team. The sample was composed of the observation of 1137 attacks in 21 games of the 2015-2016 Brazilian women's Volleyball League champion team. The results were significant to identify the predictive factors of the attack efficacy against high $\left(x^{2}=110.562 ; p<0.00001\right)$, intermediate $\left(x^{2}=64.134 ; p<0.00001\right)$ and low $\left(x^{2}=62.137 ; p<0.00001\right)$ performance opponents. Thus, it is concluded that when playing against high- performance teams, powerful attack for position 6 and second attack tempo increase the chances of attack point; powerful attacks for position 1 and 5 reduce the chances of game continuation; and powerful attack for position 6 increases the chances of blocking. In addition, in games against intermediate-performance teams, reception that allows organized attack without all attack options reduces the chances of attack point and game continuation, while the reception effect did not seem to be a predictive factor of the attack efficacy in games against high- and low-performance opponents and, finally, in games against low-performance teams, powerful attack for position 1 reduces the chances of game continuation and attack blocking.

Key words: Efficacy; Performance; Volleyball.

Resumo - Objetivou-se identificar os fatores preditivos da eficácia do ataque da equipe campeã da superliga feminina conforme o nivel de desempenho das equipes adversárias. A amostra compôs-se pela observação de 1137 ataques ocorridos em 21 jogos da equipe campeã da Superliga Feminina 2015-2016. Os resultados mostraram-se significativos na identificação dos fatores preditivos da eficácia do ataque em confrontos contra adversários de desempenho elevado $\left(x^{2}=110,562 ; p<0,00001\right)$, intermediário $\left(x^{2}=64,134 ; p<0,00001\right)$ e baixo $\left(x^{2}=62,137 ; p<0,00001\right)$. Assim, conclui-se que ao enfrentar equipes de elevado desempenho o ataque potente para a posição 6 e o $2^{\circ}$ tempo de ataque aumentaram as chances de pontuar no ataque; os ataques potentes para a posição 1 e 5 reduziram as chances de ocorrer a continuidade do jogo e o ataque potente para a posiçẫo 6 aumentaram as chances de ocorrer o bloqueio. Além disso, em jogos contra equipes de desempenho intermediário a recepção que permitiu o ataque organizado sem todas as opçôes de ataque reduziu as chances de pontuar no ataque e de continuidade do jogo, enquanto que a eficácia da recepção não mostrou-se como fator preditivo da eficácia do ataque em jogos contra adversários de elevado e baixo desempenho; e, finalmente, em jogos contra equipes de baixo desempenho o ataque potente para a posição 1 reduziu as chances de ocorrer a continuidade do jogo e o bloqueio do ataque.

Palavras-chave: Desempenho; Eficácia; Voleibol.
1 Universidade Federal de Goiás. Goiânia, GO. Brazil.

2 Centro Universitário UNA de Belo Horizonte. Belo Horizonte, MG. Brazil.

3 Universidade Federal de Minas Gerais. Belo Horizonte, MG. Brazil.

4 Centro Universitário Estácio de Brasília. Taguatinga, DF. Brazil.

Received: September 25, 2018 Accepted: April 06, 2019

How to cite this article Rocha ACR, Freire AB, Martins LR, Maia MP, Guimarães JS, Ugrinowitsch $\mathrm{H}$, Castro HO, Costa GDCT. Predictive factors of the attack efficacy: the case of the Brazilian women's Volleyball League champion team. Rev Bras Cineantropom Desempenho Hum 2019, 21:e59383. DOI: http://dx.doi.org/10.1590/19800037.2019v21e59383

Copyright: This work is licensed under a Creative Commons Attribution 4.0 International License. 


\section{INTRODUCTION}

Notational analysis in sports is not new ${ }^{1,2}$ and allows understanding which aspects of the game influence sports performance ${ }^{3,4}$. In the case of volleyball, it is observed that, in males, attack efficacy is predicted by excellent receptions that allow organized attack with all attack options ${ }^{5}$. In addition, $1^{\text {st }}$ and $2^{\text {nd }}$ attack tempo $0^{6,7}$ and powerful parallel and diagonal attacks ${ }^{8,9}$ predict attack efficacy in both sexes. However, analysis regarding sex shows that there are differences in the type of game played, derived from the power used in attacks, as well as the speed of the female game ${ }^{10,11}$, that is, in women's volleyball, attack has lower power and the game is slower compared to men's volleyball.

In analyzing the attack efficacy in complex $1^{12-14}$, that is, in the game procedures regarding reception, setting and attack, it is verified that the result of the reception action in men's volleyball ${ }^{6,13}$, as well as the attack tempo ${ }^{13,15}$ and attack type $\mathrm{e}^{10,13,16}$ in both sexes, influence the attack efficacy and that the type of game practiced changes according to the level of performance of the opposing team ${ }^{12}$.

Thus, the quality of the opposing team enables managing risks in decision-making ${ }^{17}$, and the quality of the game results in changes in the type of game practiced ${ }^{12}$, suggesting that situational constraints must be taken into account when analyzing volleyball game ${ }^{18,19}$. In this context, there is a gap in literature, since most studies had been devoted to the comprehension of the game in a generalized manner ${ }^{12,13,20}$, but the present research aimed to identify the predictive factors of the attack efficacy of the Brazilian women's League champion team according to the level of performance of the opposing team, indicated by the final classification of teams.

\section{METHOD}

\section{Sample}

The convenience sample consisted of the observation of 1137 attacks in 21 games of the 2015-2016 Brazilian women's Volleyball League champion team, of the total of 27 games played in that season. This sample is relevant since the Brazilian women's volleyball is internationally ranked among the top four teams and most of its players participate in the Brazilian Volleyball League.

\section{Variables}

Level of performance: for the analysis of this variable, teams were divided according to classification at the end of the championship. Thus, teams that finished competition between the $1^{\text {st }}$ and $4^{\text {th }}$ positions were considered high performance, teams that finished competition between the $5^{\text {th }}$ and $8^{\text {th }}$ positions were considered intermediate performance and those that finished competition between the $9^{\text {th }}$ and the $12^{\text {th }}$ positions were considered low performance.

Reception Efficacy: reception efficacy was analyzed using the instrument proposed by Maia and Mesquita ${ }^{20}$ : 
- Poor reception (C): reception that did not allow organized attack.

- Moderate Reception (B): Reception that allowed organized attack, although not all attackers were available for the attack.

- Excellent reception (A): reception that allowed organized attack with all attackers available for the attack.

Attack tempo: for this analysis, the instrument proposed by Afonso et al. ${ }^{15}$ was used:

- $1^{\text {st }}$ attack tempo: the attacker jumped during or immediately after setting, in which one step can occur after setting;

- $2^{\text {nd }}$ attack tempo: the attacker made two or three steps after setting;

- $3^{\text {rd }}$ attack tempo: the attacker waited for the ball to reach the peak of the upward trajectory and only then began the attack step.

Attack type: attack type was analyzed according to instrument proposed by Costa et al. ${ }^{21}$. Attacks exploiting block, when carried out with power, were grouped into powerful attacks on the parallel or diagonal, while Off-speed Attack that exploited block were grouped into the Offspeed Attack category:

- Powerful for position 1 (APP1): Attack carried on downward trajectory towards position 1.

- Powerful for position 6 (APP6): Attack carried on downward trajectory towards position 6 .

- Powerful for position 5 (APP5): Attack carried on downward trajectory towards position 5 .

- Powerful for position 4 (APP4): Attack carried on downward trajectory towards position 4 .

- Powerful for position 3 (APP3): Attack carried on downward trajectory towards position 3 .

- Powerful for position 2 (APP3): Attack carried on downward trajectory towards position 2 .

- Off-speed Attack (OSA): Attack performed against the ball with less force.

Number of blockers: Corresponds to the number of blockers that opposes the opposing attack. Thus, the following categories were obtained:

- No block: corresponds to the absence of blockers due to the excellence of setting.

- Single block (1x1): corresponds to the block of only one player.

- Double broken block $(1+1 \times 1)$ : corresponds to the non-compacted block of two players.

- Double compact block (2x1): corresponds to the block of two players, either compacted or not.

- Triple broken block $(2+1 \mathrm{x} 1)$ : corresponds to the non-compacted block 
of three players.

- Triple compacted block (3x1): corresponds to the compacted block of three players.

Attack efficacy: to analyze attack efficacy, the instrument proposed by Marcelino, Mesquita and Sampaio ${ }^{22}$ was used, obtaining the following categories:

- Error: attacker failed in the attack by striking the ball in the net, out or some infraction was made.

- Block: attacker failed in attack due to opponent block

- Continuation: the attack action did not result in a terminal action and allowed the opponent counterattack.

- Point: The attack resulted in a direct point.

\section{Data collection procedure}

All games were recorded from the top perspective, i.e., about 7-9 meters behind the bottom line of the court and the camera was positioned approximately three meters above ground level for better viewing of video scenes. A Sony camera with $1080 \mathrm{p} \mathrm{HD}$ resolution and $60 \mathrm{~Hz}$ frequency was used for data collection. Observers were physical education professionals with at least 5-year experience in the function of observational analyzers. For the reliability calculation, $20 \%$ of actions were re-analyzed, exceeding the reference value of $10 \%{ }^{23}$. Cohen's Kappa values for inter- and intra-observer reliability, respectively, were: reception efficacy $=0.98$ and 0.98 ; attack tempo $=0.96$ and 0.94 ; attack type $=1.00$ and 1.00 ; attack efficacy $=1.00$ and 1.00 . In this sense, the reliability values are above the reference value of $0.75^{24}$.

\section{Statistical Procedures}

Descriptive analysis and multinominal logistic analysis were performed, observing the relationship of independent variables with the dependent variable one by one. In this context, attack efficacy was considered as dependent variable and attack efficacy, attack tempo and attack type were independent variables, since the number of blockers was not associated with attack efficacy. In order to avoid inconsistencies in the prediction model due to the low occurrence, APP 2 and APP4 attacks were excluded. In addition, the analysis of the predictive attack factors was stratified according to the level of performance of opponents, obtaining an analysis for each of them. In data treatment, $5 \%$ significance level $(\mathrm{p} \leq 0.05)$ was adopted and SPSS software version 20.0 was used.

\section{RESULTS}

The descriptive analysis is presented in table 1 and showed that the occurrences of game procedures varied, although the distribution tendency modified little according to the level of performance of the opposing team. 
Table 1. Descriptive analysis of game procedures in relation to the performance of the opposing team

\begin{tabular}{|c|c|c|c|c|c|c|}
\hline \multirow{2}{*}{\multicolumn{3}{|c|}{ Game procedures }} & \multicolumn{3}{|c|}{ Level of performance } & \multirow{3}{*}{$\begin{array}{l}\text { Total } \\
161\end{array}$} \\
\hline & & & \multirow{2}{*}{$\begin{array}{c}\text { High } \\
76\end{array}$} & \multirow{2}{*}{$\begin{array}{c}\text { Intermediate } \\
49\end{array}$} & \multirow{2}{*}{$\begin{array}{c}\text { Low } \\
36\end{array}$} & \\
\hline \multirow{6}{*}{ 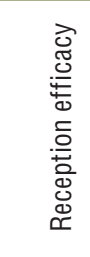 } & \multirow{2}{*}{ Reception C } & Occurred & & & & \\
\hline & & $\%$ Occurred & $47.2 \%$ & $30.4 \%$ & $22.4 \%$ & $100.0 \%$ \\
\hline & \multirow{2}{*}{ Reception B } & Occurred & 147 & 70 & 34 & 251 \\
\hline & & $\%$ Occurred & $58.6 \%$ & $27.9 \%$ & $13.5 \%$ & $100.0 \%$ \\
\hline & \multirow{2}{*}{ Reception A } & Occurred & 337 & 248 & 140 & 725 \\
\hline & & $\%$ Occurred & $46.5 \%$ & $34.2 \%$ & $19.3 \%$ & $100.0 \%$ \\
\hline \multirow{6}{*}{ 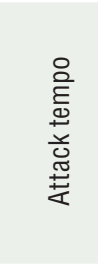 } & \multirow{2}{*}{$1^{\text {st }}$ tempo } & Occurred & 107 & 59 & 43 & 209 \\
\hline & & $\%$ Occurred & $51.2 \%$ & $28.2 \%$ & $20.6 \%$ & $100.0 \%$ \\
\hline & \multirow{2}{*}{$2^{\text {nd }}$ tempo } & Occurred & 412 & 276 & 142 & 830 \\
\hline & & $\%$ Occurred & $49.6 \%$ & $33.3 \%$ & $17.1 \%$ & $100.0 \%$ \\
\hline & \multirow{2}{*}{$3^{\text {rd }}$ tempo } & Occurred & 41 & 32 & 25 & 98 \\
\hline & & $\%$ Occurred & $41.8 \%$ & $32.7 \%$ & $25.5 \%$ & $100.0 \%$ \\
\hline \multirow{12}{*}{ 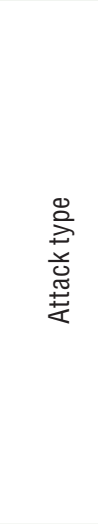 } & \multirow{2}{*}{ APP1 } & Occurred & 174 & 84 & 35 & 293 \\
\hline & & $\%$ Occurred & $59.4 \%$ & $28.7 \%$ & $11.9 \%$ & $100.0 \%$ \\
\hline & \multirow{2}{*}{ APP6 } & Occurred & 120 & 70 & 48 & 238 \\
\hline & & $\%$ Occurred & $50.4 \%$ & $29.4 \%$ & $20.2 \%$ & $100.0 \%$ \\
\hline & \multirow{2}{*}{ APP5 } & Occurred & 145 & 88 & 45 & 278 \\
\hline & & $\%$ Occurred & $52.2 \%$ & $31.7 \%$ & $16.2 \%$ & $100.0 \%$ \\
\hline & \multirow{2}{*}{ APP4 } & Occurred & 5 & 7 & 7 & 19 \\
\hline & & $\%$ Occurred & $26.3 \%$ & $36.8 \%$ & $36.8 \%$ & $100.0 \%$ \\
\hline & \multirow{2}{*}{ APP2 } & Occurred & 3 & 4 & 9 & 16 \\
\hline & & $\%$ Occurred & $18.8 \%$ & $25.0 \%$ & $56.3 \%$ & $100.0 \%$ \\
\hline & \multirow{2}{*}{ OSA } & Occurred & 113 & 114 & 66 & 293 \\
\hline & & $\%$ Occurred & $38.6 \%$ & $38.9 \%$ & $22.5 \%$ & $100.0 \%$ \\
\hline \multirow{14}{*}{ 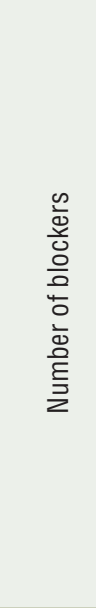 } & \multirow{2}{*}{ No block } & Occurred & 4 & 1 & 2 & 7 \\
\hline & & $\%$ Occurred & $57.1 \%$ & $14.3 \%$ & $28.6 \%$ & $100.0 \%$ \\
\hline & \multirow{2}{*}{ Single block Simples } & Occurred & 107 & 88 & 65 & 260 \\
\hline & & $\%$ Occurred & $41.2 \%$ & $33.8 \%$ & $25.0 \%$ & $100.0 \%$ \\
\hline & \multirow{2}{*}{ Block 1+1 } & Occurred & 138 & 111 & 39 & 288 \\
\hline & & $\%$ Occurred & $47.9 \%$ & $38.5 \%$ & $13.5 \%$ & $100.0 \%$ \\
\hline & \multirow{2}{*}{ Double block } & Occurred & 287 & 131 & 82 & 500 \\
\hline & & $\%$ Occurred & $57.4 \%$ & $26.2 \%$ & $16.4 \%$ & $100.0 \%$ \\
\hline & Block 2+1 & Occurred & 8 & 8 & 4 & 20 \\
\hline & DIUCK $\angle+1$ & $\%$ Occurred & $40.0 \%$ & $40.0 \%$ & $20.0 \%$ & $100.0 \%$ \\
\hline & Trinle hlock & Occurred & 4 & 16 & 13 & 33 \\
\hline & IIIPE DIUCh & $\%$ Occurred & $12.1 \%$ & $48.5 \%$ & $39.4 \%$ & $100.0 \%$ \\
\hline & No block reauired & Occurred & 12 & 12 & 5 & 29 \\
\hline & TNO DIock required & $\%$ Occurred & $41.4 \%$ & $41.4 \%$ & $17.2 \%$ & $100.0 \%$ \\
\hline
\end{tabular}

The analysis of predictive factors of the attack efficacy in confrontations against high-performance opponents was statistically significant $\left(\mathrm{x}^{2}\right.$ $=110.562 ; \mathrm{p}<0.00001)$, as shown in table 2 .

The analysis of predictive factors of the attack efficacy in confrontations against intermediate-performance opponents was statistically significant $\left(\mathrm{x}^{2}=64,134 ; \mathrm{p}<0.00001\right)$, as shown in table 3 .

The analysis of predictive factors of the attack efficacy in confrontations against low-performance opponents was statistically significant $\left(\mathrm{x}^{2}\right.$ $=62.137 ; \mathrm{p}<0.00001)$, as shown in table 4 . 
Table 2. Predictive factors of the attack efficacy against high-performance opponents

\begin{tabular}{|c|c|c|c|c|c|c|c|c|}
\hline \multirow{2}{*}{\multicolumn{2}{|c|}{ Attack efficacy a }} & \multirow{4}{*}{$\begin{array}{l}\% \\
8.70 \%\end{array}$} & \multirow{4}{*}{$\begin{array}{c}\begin{array}{c}\text { Crude Odds } \\
\text { Ratio }\end{array} \\
.353\end{array}$} & \multirow{4}{*}{$\begin{array}{c}\text { Crude } p \\
0.021\end{array}$} & \multirow{4}{*}{$\begin{array}{c}\text { Adjusted } \\
\text { Odds Ratio } \\
1.065\end{array}$} & \multirow{4}{*}{$\begin{array}{c}\text { Adjusted } p \\
.931\end{array}$} & \multirow{2}{*}{\multicolumn{2}{|c|}{$\begin{array}{l}95 \% \text { confidence interval for } \\
\operatorname{Exp}(B)\end{array}$}} \\
\hline & & & & & & & & \\
\hline \multirow{11}{*}{$\begin{array}{l}\frac{r}{0} \\
\frac{0}{\infty}\end{array}$} & & & & & & & & \\
\hline & Reception C & & & & & & .255 & 4.448 \\
\hline & Reception B & $37.70 \%$ & .746 & 0.487 & 2.593 & .060 & .960 & 7.002 \\
\hline & Reception $A^{b}$ & $53.60 \%$ & & & & & & \\
\hline & $1^{\text {st }}$ tempo & $29.00 \%$ & 4.190 & 0.021 & 3.980 & .131 & .663 & 23.894 \\
\hline & $2^{\text {nd }}$ tempo & $65.20 \%$ & 2.886 & 0.046 & 2.016 & .379 & .423 & 9.616 \\
\hline & $3^{\text {rd }}$ tempo $^{b}$ & $5.80 \%$ & & & & & & \\
\hline & APP1 & $33.30 \%$ & 0.397 & 0.059 & .725 & 0.618 & .205 & 2.56 \\
\hline & APP6 & $34.80 \%$ & 1.875 & 0.285 & 13.614 & $.025^{*}$ & 1.383 & 134.030 \\
\hline & APP5 & $18.80 \%$ & 0.539 & 0.213 & .514 & .328 & .136 & 1.949 \\
\hline & $\mathrm{OSA}^{\mathrm{b}}$ & $13.00 \%$ & & & & & & \\
\hline \multirow{10}{*}{ 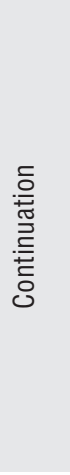 } & Reception C & $18.10 \%$ & .605 & 0.152 & 1.341 & .612 & .432 & 4.165 \\
\hline & Reception B & $27.40 \%$ & .726 & 0.379 & 1.419 & .445 & .578 & 3.481 \\
\hline & Reception $\mathrm{A}^{\mathrm{b}}$ & $54.40 \%$ & & & & & & \\
\hline & $1^{\text {st }}$ tempo & $15.80 \%$ & 1.222 & 0.682 & 1.080 & .917 & .560 & 4.552 \\
\hline & $2^{\text {nd }}$ tempo & $71.20 \%$ & 1.599 & 0.214 & 1.415 & .566 & .432 & 4.636 \\
\hline & $3^{\text {rd }}$ tempo ${ }^{b}$ & $13.00 \%$ & & & & & & \\
\hline & APP1 & $20.00 \%$ & 0.141 & 0.0001 & .134 & $.0001^{*}$ & .047 & .384 \\
\hline & APP6 & $17.70 \%$ & .467 & 0.1580 & 2.344 & .115 & .264 & 20.818 \\
\hline & APP5 & $25.10 \%$ & 0.218 & 0.0001 & .232 & $.008^{*}$ & .080 & .679 \\
\hline & $\mathrm{OSA}^{\mathrm{b}}$ & $37.20 \%$ & & & & & & \\
\hline \multirow{10}{*}{ 릉 } & Reception C & $10.60 \%$ & .222 & 0.0001 & 1.082 & .889 & .356 & 3.293 \\
\hline & Reception B & $25.40 \%$ & .639 & 0.2120 & 1.276 & .585 & .533 & 3.058 \\
\hline & Reception $\mathrm{A}^{\mathrm{b}}$ & $64.00 \%$ & & & & & & \\
\hline & $1^{\text {st }}$ tempo & $17.40 \%$ & 8.119 & 0.0001 & 4.075 & .062 & .929 & 17.863 \\
\hline & $2^{\text {nd }}$ tempo & $79.40 \%$ & 7.996 & 0.0001 & 5.134 & $.011^{*}$ & 1.463 & 18.017 \\
\hline & $3^{\text {rd }}$ tempo $^{b}$ & $3.20 \%$ & & & & & & \\
\hline & APP1 & $35.00 \%$ & 0.816 & 0.617 & .735 & .568 & .255 & 2.119 \\
\hline & APP6 & $25.40 \%$ & 2.011 & 0.193 & 10.239 & $.037^{\star}$ & 1.150 & 91.195 \\
\hline & APP5 & $28.00 \%$ & .757 & 0.511 & .816 & .715 & .274 & 2.430 \\
\hline & OSA ${ }^{b}$ & $11.60 \%$ & & & & & & \\
\hline
\end{tabular}

Note. ${ }^{a}$ The reference category for the dependent variable is the attack error; ${ }^{\mathrm{b}}$ The reference category for the independent variable; * difference for $p<0.05$

Table 3. Predictive factors of the attack efficacy against intermediate-performance opponents

\begin{tabular}{|c|c|c|c|c|c|c|c|c|}
\hline \multirow{2}{*}{\multicolumn{2}{|c|}{ Attack efficacy a }} & \multirow{3}{*}{$\begin{array}{l}\% \\
31.20 \%\end{array}$} & \multirow{3}{*}{$\begin{array}{c}\text { Crude Odds } \\
\text { Ratio } \\
0.458\end{array}$} & \multirow{3}{*}{$\begin{array}{c}\text { Crude } p \\
.064\end{array}$} & \multirow{3}{*}{$\begin{array}{c}\begin{array}{c}\text { Adjusted } \\
\text { Odds Ratio }\end{array} \\
.265\end{array}$} & \multirow{3}{*}{$\begin{array}{c}\text { Adjusted } p \\
.317\end{array}$} & \multicolumn{2}{|c|}{$\begin{array}{l}95 \% \text { confidence interval for } \\
\operatorname{Exp}(B)\end{array}$} \\
\hline & & & & & & & Lower limit & Upper limit \\
\hline \multirow{10}{*}{$\begin{array}{l}\text { 흠 } \\
\frac{0}{\infty}\end{array}$} & Reception C & & & & & & .020 & 3.565 \\
\hline & Reception B & $37.50 \%$ & 1.038 & .915 & .211 & .057 & .042 & 1.050 \\
\hline & Reception $\mathrm{A}^{\mathrm{b}}$ & $31.30 \%$ & & & & & & \\
\hline & $1^{\text {st }}$ tempo & $22.60 \%$ & 3.556 & 0.020 & 1.706 & .749 & .065 & 44.970 \\
\hline & $2^{\text {nd }}$ tempo & $67.70 \%$ & 2.299 & 0.077 & 1.671 & .714 & .107 & 26.055 \\
\hline & $3^{\text {rd }}$ tempo $^{b}$ & $9.70 \%$ & & & & & & \\
\hline & APP1 & $12.90 \%$ & .417 & .058 & .825 & .845 & .121 & 5.629 \\
\hline & APP6 & $35.50 \%$ & 2.847 & 0.070 & 3.048 & .257 & .443 & 20.977 \\
\hline & APP5 & $29.00 \%$ & 0.417 & .061 & .871 & .865 & .178 & 4.255 \\
\hline & $\mathrm{OSA}^{\mathrm{b}}$ & $22.60 \%$ & & & & & & \\
\hline
\end{tabular}




\begin{tabular}{|c|c|c|c|c|c|c|c|c|}
\hline \multirow{2}{*}{\multicolumn{2}{|c|}{ Attack efficacy a }} & \multirow[t]{2}{*}{$\%$} & \multirow{2}{*}{$\begin{array}{l}\text { Crude Odds } \\
\quad \text { Ratio }\end{array}$} & \multirow[t]{2}{*}{ Crude $p$} & \multirow{2}{*}{$\begin{array}{l}\text { Adjusted } \\
\text { Odds Ratio }\end{array}$} & \multirow[t]{2}{*}{ Adjusted $p$} & \multicolumn{2}{|c|}{$\begin{array}{l}\text { 95\% confidence interval for } \\
\operatorname{Exp}(B)\end{array}$} \\
\hline & & & & & & & Lower limit & Upper limit \\
\hline \multirow{10}{*}{ 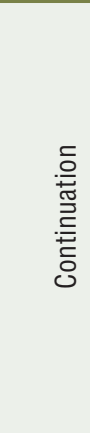 } & Reception C & $19.10 \%$ & 0.837 & .578 & .450 & .468 & .052 & 3.873 \\
\hline & Reception B & $17.00 \%$ & 0.778 & .409 & .172 & $.010^{*}$ & .045 & .661 \\
\hline & Reception $A^{b}$ & $63.40 \%$ & & & & & & \\
\hline & $1^{\text {st }}$ tempo & $11.30 \%$ & 1.000 & 1.000 & 1.217 & .890 & .075 & 19.410 \\
\hline & $2^{\text {nd }}$ tempo & $75.90 \%$ & 1.374 & 0.352 & 2.856 & .351 & .315 & 25.919 \\
\hline & $3^{\text {rd }}$ tempo $^{b}$ & $12.80 \%$ & & & & & & \\
\hline & APP1 & $15.60 \%$ & 0.144 & .001 & .458 & .3240 & .097 & 2.159 \\
\hline & APP6 & $13.50 \%$ & 0.608 & .353 & .506 & .446 & .088 & 2.919 \\
\hline & APP5 & $22.00 \%$ & 0.199 & .001 & .289 & .0630 & .078 & 1.070 \\
\hline & OSA ${ }^{b}$ & $48.90 \%$ & & & & & & \\
\hline \multirow{10}{*}{ 昱 } & Reception C & $7.10 \%$ & 0.345 & .001 & .231 & .190 & .026 & 2.064 \\
\hline & Reception B & $20.20 \%$ & 0.672 & .180 & .217 & $.023^{*}$ & .058 & .807 \\
\hline & Reception $A^{b}$ & $72.60 \%$ & & & & & & \\
\hline & $1^{\text {st }}$ tempo & $18.50 \%$ & 5.091 & 0.0001 & 3.054 & .440 & .179 & 51.948 \\
\hline & $2^{\text {nd }}$ tempo & $78.00 \%$ & 5.378 & 0.0001 & 4.343 & .215 & .426 & 44.261 \\
\hline & $3^{\text {rd }}$ tempo $^{b}$ & $3.50 \%$ & & & & & & \\
\hline & APP1 & $32.70 \%$ & .747 & 0.456 & 2.262 & .299 & .485 & 10.555 \\
\hline & APP6 & $22.60 \%$ & 2.739 & 0.059 & 2.135 & .395 & .371 & 12.273 \\
\hline & APP5 & $25.00 \%$ & .672 & .315 & .803 & .746 & .214 & 3.019 \\
\hline & OSA & $19.70 \%$ & & & & & & \\
\hline
\end{tabular}

Note. a The reference category for the dependent variable is the attack error; $b$ The reference category for the independent variable; * difference for $p<0.05$

Table 4. Predictive factors of attack efficacy against low-performance opponents

\begin{tabular}{|c|c|c|c|c|c|c|c|c|}
\hline \multirow{2}{*}{\multicolumn{2}{|c|}{ Attack efficacy a }} & \multirow[t]{2}{*}{$\%$} & \multirow[t]{2}{*}{ Continue } & \multirow[t]{2}{*}{ Crude $p$} & \multirow{2}{*}{$\begin{array}{l}\text { Adjusted } \\
\text { Odds Ratio }\end{array}$} & \multirow[t]{2}{*}{ Adjusted $p$} & \multicolumn{2}{|c|}{$\begin{array}{l}\text { 95\% confidence interval for } \\
\operatorname{Exp}(B)\end{array}$} \\
\hline & & & & & & & Lower limit & Upper limit \\
\hline \multirow{10}{*}{$\begin{array}{l}\text { 흠 } \\
\frac{\text { 음 }}{n}\end{array}$} & Reception C & $15.80 \%$ & .075 & .299 & .413 & .406 & .051 & 3.321 \\
\hline & Reception B & $21.10 \%$ & .806 & .837 & .568 & .474 & .121 & 2.673 \\
\hline & Reception $A^{b}$ & $63.20 \%$ & & & & & & \\
\hline & $1^{\text {st }}$ tempo & $26.30 \%$ & 4.000 & .121 & 1.454 & .778 & .108 & 19.566 \\
\hline & $2^{\text {nd }}$ tempo & $63.20 \%$ & 3.667 & .101 & 1.807 & .607 & .190 & 17.175 \\
\hline & $3^{\text {rd }}$ tempo $^{b}$ & $10.50 \%$ & & & & & & \\
\hline & APP1 & $5.30 \%$ & .083 & 0.032 & .083 & $.035^{\star}$ & .008 & .844 \\
\hline & APP6 & $31.60 \%$ & .833 & .820 & .921 & .930 & .147 & 5.767 \\
\hline & APP5 & $21.10 \%$ & .333 & 0.153 & .318 & .151 & .067 & 1.517 \\
\hline & $\mathrm{OSA}^{\mathrm{b}}$ & $42.10 \%$ & & & & & & \\
\hline \multirow{10}{*}{ 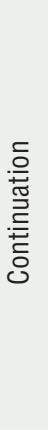 } & Reception C & $23.70 \%$ & .516 & 0.180 & .278 & .142 & .050 & 1.536 \\
\hline & Reception B & $13.20 \%$ & 1.053 & .933 & .554 & .380 & .148 & 2.073 \\
\hline & Reception $A^{b}$ & $63.20 \%$ & & & & & & \\
\hline & $1^{\text {st }}$ tempo & $13.20 \%$ & .966 & .957 & .479 & .490 & .059 & 3.866 \\
\hline & $2^{\text {nd }}$ tempo & $67.10 \%$ & 1.841 & .249 & 1.230 & .816 & .215 & 7.028 \\
\hline & $3^{\text {rd }}$ tempo $^{b}$ & $19.70 \%$ & & & & & & \\
\hline & APP1 & $14.50 \%$ & .012 & 0.001 & .120 & $.001^{*}$ & .033 & .444 \\
\hline & APP6 & $15.80 \%$ & .212 & 0.032 & .280 & .143 & .051 & 1.536 \\
\hline & APP5 & $17.10 \%$ & .138 & 0.001 & .141 & .003 & .039 & .505 \\
\hline & $\mathrm{OSA}^{\mathrm{b}}$ & $52.60 \%$ & & & & & & \\
\hline
\end{tabular}




\begin{tabular}{|c|c|c|c|c|c|c|c|c|}
\hline \multirow{2}{*}{\multicolumn{2}{|c|}{ Attack efficacy a }} & \multirow[t]{2}{*}{$\%$} & \multirow{2}{*}{ Continue } & \multirow[t]{2}{*}{ Crude $p$} & \multirow{2}{*}{$\begin{array}{c}\text { Adjusted } \\
\text { Odds Ratio }\end{array}$} & \multirow[t]{2}{*}{ Adjusted $p$} & \multicolumn{2}{|c|}{$\begin{array}{c}95 \% \text { confidence interval for } \\
\operatorname{Exp}(B)\end{array}$} \\
\hline & & & & & & & Lower limit & Upper limit \\
\hline \multirow{10}{*}{.등 } & Reception C & $9.40 \%$ & .178 & 0.002 & .247 & .112 & .044 & 1.387 \\
\hline & Reception B & $12.90 \%$ & .815 & .740 & .569 & .403 & .152 & 2.134 \\
\hline & Reception $A^{b}$ & $77.60 \%$ & & & & & & \\
\hline & $1^{\text {st }}$ tempo & $29.40 \%$ & 6.171 & .013 & 1.602 & .669 & .184 & 13.931 \\
\hline & $2^{\text {nd }}$ tempo & $64.70 \%$ & 7.929 & 0.001 & 3.016 & .239 & .480 & 18.951 \\
\hline & $3^{\text {rd }}$ tempo $^{b}$ & $5.90 \%$ & & & & & & \\
\hline & APP1 & $21.20 \%$ & .414 & 0.145 & .382 & .138 & .107 & 1.364 \\
\hline & APP6 & $30.60 \%$ & 0.996 & .996 & 1.010 & .991 & .194 & 5.256 \\
\hline & APP5 & $27.10 \%$ & .529 & 0.285 & .451 & .207 & .131 & 1.552 \\
\hline & $\mathrm{OSA}^{\mathrm{b}}$ & $21.20 \%$ & & & & & & \\
\hline
\end{tabular}

Note. a The reference category for the dependent variable is the attack error; $b$ The reference category for the independent variable; * difference for $p<0.05$

\section{DISCUSSION}

The aim of the present study was to identify the predictive factors of the attack efficacy of the Brazilian women's League champion team according to the level of performance of the opposing team. Descriptive analysis showed that, regardless of level performance of the opposing team, there was higher occurrence of A reception, second attack tempo, powerful attack for position 1 and double compact block. These results corroborate literature, which shows greater occurrence of receptions that allow organized attack ${ }^{8,12,21}$, second attack tempo ${ }^{8,9,13,25}$, powerful attack ${ }^{13}$ and double block $^{26}$. In this context, it was observed that the offensive game pattern of the Brazilian League champion team did not change according to the level of performance of the opponent and therefore, to a certain extent, do not corroborate results that indicate that the quality of the opposing team and the quality of confrontation enable managing risks in decision-making ${ }^{17}$, as well as changes in the type of game practiced ${ }^{12}$. Thus, it is suggested that the complex I, composed of reception, setting and attack, suffered little influence from the opposing team when considering the game procedures analyzed. However, these results do not allow inferring about the offensive strategy used, a factor that can contribute to similarities observed in the descriptive analysis and that, according to Ramos et al. ${ }^{19}$, changes according to the opponent and moment of the game.

Analysis of predictive factors of the attack efficacy on games against high-performance teams showed that powerful attack for position 6 and second attack tempo increased the chances of attack point; powerful attacks for positions 1 and 5 reduced the chances of game continuation; and powerful attack for position 6 increased the chances of blocking. These results partially corroborate literature, since $1^{\text {st }}$ and $2^{\text {nd }}$ attacks tempo are predictive factors of the attack point, as well as the powerful attacks ${ }^{8,11,27}$. Regarding block, there is partial agreement with the study by Conti et al. ${ }^{9}$, who demonstrated increased chances of blocking after powerful attacks 
performed with parallel and diagonal. In this way, it was observed that to set faster, specifically in second attack tempo and powerful attack are essential in high-performance women's volleyball. However, the fact that the powerful attack for position 6 has increased the chances of block to occur suggests greater concern with tactical procedures to protect this type of attack by the attacking team. Analysis of predictive factors of the attack efficacy on games against intermediate-performance teams showed that reception $\mathrm{B}$ reduced the chances of attack point and game continuation. The results corroborate the study by Conti et al. ${ }^{9}$, who demonstrated reduction of the chances of point after receptions that do not allow attack with all attack options. In addition, the study by Costa et al. ${ }^{10}$ demonstrated that defeat on the set is predicted by non-scoring attacks and poor quality receptions. Thus, it is possible to suggest that receptions that do not allow attack organization with all attack options limit distribution and, consequently, restrict the action of the attacker, making difficult obtaining the point.

Analysis of predictive factors of the attack efficacy on games against low-performance teams showed that powerful attack for position 1 reduced the chances of game continuation and attack block. These results partially corroborate the study by Costa et al. ${ }^{8}$, who demonstrated reduction of game continuation after powerful attacks performed for position 1 . On the other hand, the results disagree with those obtained by Conti et al. ${ }^{9}$, who observed an increase in the chances of game continuation occurring after powerful attack for position 1. Although there are divergences in the predictive factors pointed out in the present research, it was observed that this fact can be related to the specific analysis of the aforementioned studies, since they analyzed attacks made from positions 4, 2 and 1 of the men's national league. However, since literature points out that the game played in women's volleyball is supportive, that is, it consists of a greater amount of defense than the game played in men's volleyball ${ }^{6,12,28}$, it can be suggested that attacks made from position 1 aim to limit the offensive construction, considering that the setter occupies such a position in the defensive system when in the defense zone.

The present study did not show reception efficacy as a predictive factor of the attack efficacy in games against high- and low-performance opponents, and did not show the $1^{\text {st }}$ attack tempo as a predictive factor of the attack efficacy, regardless of opponent's level of performance. These results contradict the available literature in both sexes ${ }^{9,14,26,29}$, which demonstrated the influence of reception efficacy on the attack efficacy, as well as the $1^{\text {st }}$ attack tempo on point achievement. According to the Brazilian Volleyball Confederation $(\mathrm{CBV})^{30}$, the efficacy of attackers of the champion team was higher than the average of the competition and 3 attackers of the champion team were ranked among the 10 most effective attackers of the competition, which suggests that when playing against high-performance opponents, high-quality receptions are a prerequisite and teams should have specific offensive tactical strategies based on situational constraints. On the other hand, in confrontations with low-performance teams, reception 
efficacy may not be predictive due to discrepancies in the tactical-technical performance, especially in attack, a game procedure that is shown to be a determining factor for the point achievement and winning the set ${ }^{5,14}$. With regard to attack tempo, it is possible to infer that playing in $1^{\text {st }}$ attack tempo is an essential factor and that the level of performance does not change this game procedure. Thus, it was observed that high-quality receptions and fast game are fundamental for point achievement and are not differentiating factors of sport performance.

Finally, it is hoped that the information contained in the results obtained allows adaptations of the tactical concepts used by coaches in structuring specific training to improve both attack performance through training of technical and tactical actions that potentiate the attack efficacy and in a defense situation, consolidating technical-tactical actions capable of neutralizing the opponent's actions. However, the study presents as limitations the analysis of the attack efficacy as a whole, without distinguishing distinct periods of the game (e.g., differences between sets or punctuation intervals within sets), and did not consider counterattacks.

\section{CONCLUSION}

Based on the aims, applied methods and results found, the present study showed how the performance of the opposing team influences the offensive construction of the attack, that is, in complex 1 . In this way, confrontations with low- and intermediate- performance teams were influenced by the reception efficacy and attack type, respectively. On the other hand, confrontations with high-performance teams were influenced by powerful attacks for position 1 , suggesting the need to limit the opponent's offensive construction.

\section{COMPLIANCE WITH ETHICAL STANDARDS}

\section{Funding}

This research did not receive any specific grant from funding agencies in the public, commercial, or non-profit sectors. This study was funded by the authors.

\section{Ethical approval}

The protocol of this research was written in accordance with standards set by the Declaration of Helsinki.

\section{Conflict of interest statement}

The authors have no conflict of interests to declare.

\section{Author Contributions}

Conceived and designed the experiments: GDCTC. Performed the experiments: ACRR and HOC. Analyzed data: ABF, HU, ACRR, MPM and LRM. Contributed with reagents/materials/analysis tools: ACRR, MPM, LRM and JSG. Wrote the paper: ACRR, GDCTC, ABF, JSG and HOC. 


\section{REFERENCES}

1. Eom, H. J., Schutz, N. R. (1992). Statistical analysis of Volleyball team performance. Res Q Exerc Sport 1992;63(1):11-18.

2. Hughes M, Bartlett RM. The use of performance indicators in performance analysis. J Sports Sci 2002;20:739-754.

3. Barreira J, Silva CE. National teams in Women's Soccer World Cup from 1991 to 2015: participation, performance and competitiveness. J Phys Educ Sport 2016;16(3):795-799.

4. Clemente FM, Martins FML, Mendes RS, Silva F. Social network measures to match analysis in soccer: A survey. J Phys Educ Sport 2016;16(3),823-830.

5. Marcelino R, A fonso J, Moraes JC, Mesquita I. Determinants of attack players in high-level men's volleyball. Kinesiology 2014;46(2):234-241.

6. Mesquita I, Palao JM, Marcelino R, Afonso J. Performance analysis in indoor volleyball and beach volleyball. In: McGarry T, O'Donoghue P, Sampaio J, editors. Routledge Handbook of Sports Performance Analysis, pp.367-79, 2013.

7. García-de-Alcaraz A, Ortega E, Palao JM. Effect of age group on male volleyball players' technical-tactical performance profile for the spike. Int J Perform Anal Sport 2015;15(2):668-686.

8. Costa GDCT, Maia MP, Rocha ACR, Martins LR, Gemente FRF, Campos MH, et al. Association between effect of reception and game procedures in high-level Brazilian volleyball: The case of the women's "Superliga" champion team. Rev Bras Cineantropom Desempenho Hum 2017;19(6):663-675.

9. Conti G, Freire A, Evangelista B, Pedrosa G, Ugrinowitsch H, Castro H. Brazilian high level men's volleyball: characterization of the attack performed by the opposite player. Kinesiology 2018;50(2):10-17.

10. Costa GCT, Afonso J, Barbosa RV, Coutinho P, Mesquita I. Predictors of attack efficacy and attack type in high-level Brazilian women's volleyball. Kinesiology 2014;46(2):242-248.

11. Costa GDC, Barbosa RV, Freire AB, Matias CJAS, Greco PJ. Análise das estruturas do Complexo I à luz do resultado do set no voleibol feminino. Motrici 2014;10(3):40-49.

12. Costa GC, Castro HO, Evangelista BF, Malheiros LM, Greco PJ, Ugrinowitsch $\mathrm{H}$. Predicting factors of zone 4 attack in volleyball. Percep Motor Skills 2017;124(3):621-633.

13. Costa GDCT, Ceccato JS, Oliveira AS, Evangelista BFB, Castro HO, Ugrinowitsch H. Men's volleyball hight level: association between game actions on the side-out. J Phys Educ 2016;27(1):e-2755.

14. Silva M, Marcelino R, Lacerda D, João P. Match analysis in volleyball: a systematic review. Monten J Sports Sci Med 2016;5(1):35-46.

15. Afonso J, Mesquita I, Marcelino R, Silva J. Analysis of the setter's tactical action in high performance women's volleyball. Kinesiology 2010;42(1):82-89.

16. Costa G, Mesquita I, Greco PJ, Ferreira N, Moraes JC. Relação saque, recepção e ataque no voleibol juvenil masculino. Motriz: Rev Ed Fis 2010;17(1):11-18.

17. García-de-Alcaraz A, Marcelino R. Influence of match quality on men's volleyball performance at different competition levels. Int J Perform Anal Sport 2017; 17(4):394-405

18. Paulo A, Davids K, Araújo D. Co-adaptation of ball reception to the serve constrains outcomes in elite competitive volleyball. Int J Sports Sci Coach 2017;13(2):253-261.

19. Ramos A, Coutinho P, Silva P, Davids K, Guimarães E, Mesquita I. Entropy measures reveal collective tactical behaviours in volleyball teams: how variability and regularity in game actions influence competitive rankings and match status. Int J Perform Anal Sport 2017;17(6):848-862.

20. Maia N, Mesquita I. Estudo das zonas e eficácia da recepção em função do jogador recebedor no voleibol sênior feminino. Rev Bras Educ Fís Esporte 2006;20(4):257-270. 
21. Costa G, Ferreira N, Junqueira G, Afonso J, Mesquita I. Determinants of attack tactics in youth male elite volleyball. Int J Perform Anal Sport 2011;11(1):96-104.

22. Marcelino R, Mesquita I, Sampaio J. Effects of quality of opposition and match status on technical and tactical performances in elite volleyball. J Sports Sci 2011;29(7):733-741.

23. Tabachnick B, Fidell L. Using multivariate statistics. 6th ed. Boston: Allyn \& Bacon; 2013.

24. Fleiss J. Statistical methods for rates and proportions. 3rd ed. Wiley-Interscience; 2003.

25. Palao JM, Santos JÁ, Ureña A. Effect of the manner of spike execution on spike performance in volleyball. Int J Perform Anal Sport 2007;7(2):126-138.

26. Ramos A, Coutinho P, Silva P, Davids K, Mesquita I. How players exploit variability and regularity of game actions in female volleyball teams. Eur J Sport Sci 2017;17(4):473-481.

27. Stutzig N, Zimmermann B, Busch D, Siebert T. Analysis of game variables to predict scoring and performance levels in elite men's volleyball. Int J Perform Anal Sport 2015;15(3):816-829.

28. Costa GDCT, Freire A. Voleibol feminino de alto nível: análise do ataque na Superliga Feminina. Rev Bras Educ Fís Esporte 2017;31(2):365-372.

29. Silva M, Sattler T, Lacerda D, João PV. Match analysis according to the performance of team rotations in Volleyball. Int J Perform Anal Sport 2016;16(3):10761086.

30. Confederação Brasileira de Voleibol. Estatísticas por atletas - Superliga 2015-2016. Disponível em: http://superliga.cbv.com.br/15-16/index.php/2014-10-24-18-2403/estatisticas-por-atletas [04 Abr, 2019].

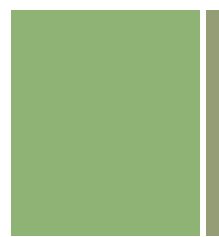

Corresponding author

Gustavo De Conti Teixeira Costa

Faculdade de Educação Física e Dança

Universidade Federal de Goiás, Campus Samambaia

Avenue Esperança s/n, Goiânia, Goiás, Brasil.

CEP: 74.690-900.

E-mail:conti02@hotmail.com 